\title{
Efficacy and learning curve of a hand-held echocardiography device in an oncology outpatient clinic: Expanding the use of echoscopic heart examination beyond cardiology
}

\author{
LEOPOLDO PÉREZ DE ISLA ${ }^{1}$, FERNANDO MORENO ${ }^{2}$, JOSE ANGEL GARCIA SAEZ ${ }^{2}$, \\ MATIAS CLAVERO $^{1}$, NUNO MORENO ${ }^{1}$, CARLOS AGUADO DE LA ROSA ${ }^{2}$, JOSE ALBERTO DE AGUSTIN ${ }^{1}$, \\ JOSE JUAN GOMEZ DE DIEGO ${ }^{1}$, MIGUEL ANGEL COBOS $^{1}$, ADRIANA SALTIJERAL ${ }^{3}$, \\ CARLOS MACAYA $^{1}$ and MIGUEL ANGEL GARCIA-FERNANDEZ ${ }^{1}$
}

Departments of ${ }^{1}$ Cardiology and ${ }^{2}$ Oncology, Clinical Hospital San Carlos, Health Research Institute, San Carlos, 28040 Madrid; ${ }^{3}$ Department of Cardiology, Hospital del Tajo, 28300 Madrid, Spain

Received October 16, 2014; Accepted March 20, 2015

DOI: $10.3892 / \mathrm{mco} .2015 .543$

\begin{abstract}
Certain chemotherapy drugs for breast cancer may induce cardiotoxicity and these patients should be echocardiographically monitored. The performance of a focused echocardiographic evaluation (echoscopy) at the patient's location by a non-cardiologist appears to be feasible. The aim of the present study was to assess the accuracy of echoscopy performed by medical oncologists in an outpatient clinic using hand-held echocardiography devices. The study cohort comprised consecutive unselected patients who attended an oncology outpatient clinic. Two medical oncologists attended a one-week training period, which included theoretical and practical teaching by an expert cardiologist. Every subject underwent two echo examinations. The first examination was performed by an oncologist using a hand-held echo device and the second was performed by a cardiologist using a 'premium' device. Out of the 101 enrolled patients, 32 were men (31.7\%) and the mean age was $56.03 \pm 16.88$ years. There was a good global agreement [intra-class correlation coefficient (ICC): 0.65 for left ventricular ejection fraction (LVEF)]. When the results were analyzed depending on the period of time when the echo studies were performed, a clear and short learning curve was observed: $\mathrm{LVEF}$ started at ICC $=0.58$ and increased to 0.66 and 0.77 in the second and third period, respectively. There were extremely few clinically significant differences and a learning curve was also evident. In conclusion, cardiac echoscopy performed by an oncologist with a hand-held
\end{abstract}

Correspondence to: Dr Leopoldo Pérez de Isla, Department of Cardiology, Clinical Hospital San Carlos, Health Research Institute, San Carlos, Profesor Martin Lagos Street s/n, 28040 Madrid, Spain E-mail: leopisla@hotmail.com

Key words: hand-held echocardiography, oncology, chemotherapy, cardiotoxicity device may lead to a similar clinical management as a study performed by an expert cardiologist with a 'premium' system in patients under chemotherapy following a short training period.

\section{Introduction}

Currently, echocardiographic departments are constantly suffering a reduction in the human resources in parallel with a significant increase in demands. Thus, the organization of these departments must be modified in order to provide the patient with the best management at the precise time. Furthermore, the patients who require an echocardiogram must move to the echo department with a consequent cost in terms of money and time (1).

Oncology is a continually growing medical branch. New drugs have been combined to delay the progression of the disease and even, to cure it. However, these may induce the development of cardiotoxicity $(2,3)$. Anthracyclines and new targeted therapies have been succeeding in breast cancer. Since anthracyclines induce an irreversible cardiotoxicity, the efforts have been directed at improving the understanding of the cardiotoxicity incidence and optimizing the measures to address the benefits of new potential cardiotoxic cancer-targeted therapies as oncological treatment. As a result, breast cancer oncologists must acknowledge the time between prescribing anthracyclines and anti-human epidermal growth factor receptor 2 therapies or the possibility of a non-anthracycline schedule to be evaluated in selected patients that would otherwise not be treated $(4,5)$. Optimal cardiac management requires a good relationship between specialists, in this case with cardiologists. The aim of this relationship must pursue the patient's benefit as the oncological prognosis should not be affected by a cardiac event that may force trastuzumab to be withdrawn. In fact, a significant improvement in cardiac examination (such as intervals and tools) and an early detection of cardiac damage with a precocious and well-directed treatment would increase the number of patients with treatment achievement (6). This is the reason why these patients should 
be echocardiographically monitored for the early detection of cardiotoxicity to adapt or even change their management $(7,8)$. The echocardiographic evaluation of these patients is targeted and it is principally focused on the left ventricular (LV) global systolic function. Furthermore, these patients have peculiar characteristics, such as the impairment in their mobility, due to the chemotherapy adverse events and immunodeficiency. Therefore, these patients become a group of individuals prone to benefit from hand-held echocardiography performed in the oncology clinic.

During the last decades, hand-held echocardiographic devices have been developed (9-11). Currently, they may carry out a complete echo study, without a significant degradation of image quality. Furthermore, the price and associated costs have considerably decreased. Thus, the performance of a focused echocardiographic evaluation (known as echoscopic heart evaluation) at the patient's location (such as the outpatient clinic and hospital room) by a non-cardiologist appears to be feasible for limited diagnostic issues (12).

The aim of the present study was to assess the accuracy of echoscopic heart evaluation performed by an oncologist with basic echocardiographic training with a simplified imaging protocol in the outpatient clinic using a hand-held device. The results of the echocardiogram performed the same day to the same patient by a cardiologist expert in cardiovascular imaging using a 'premium' device was considered the reference method. The main target variable was LV ejection fraction (LVEF).

\section{Patients and methods}

Patient population. The study cohort comprised consecutive unselected patients who attended the oncology outpatient clinic at a tertiary university hospital (Clinical Hospital San Carlos, Health Research Institute, Madrid, Spain) between October 2013 and March 2014, and had an indication to undergo a transthoracic echocardiogram. For statistical purposes, the subjects included in the study were divided into three groups, depending on the time when they were enrolled. The first third comprised patients enrolled between October 2013 and December 2013 ( 2 months). The second third comprised patients enrolled between December 2013 and January 2014 ( $\sim 2$ months) and the remaining third comprised patients between January 2014 and March 2014 ( 2 months). All the patients provided written informed consent in accordance with a protocol approved by the Institutional Review Committee.

Oncologist training. Two breast cancer oncologists attended a one-week training period that included theoretical and practical teaching by an expert cardiologist. This period focused on the evaluation of LV diameters and LVEF. Each oncologist performed 20 studies during that period. After the initial teaching period, the oncologists were able to consult the cardiologists once each patient was evaluated; however at that point, no previous measurement was modified for the study.

Echocardiography. Every subject underwent two echo examinations. The first examination was performed by one of
Table I. Main characteristics.

\begin{tabular}{lc}
\hline Characteristics & Values \\
\hline Male gender, $\mathrm{n}(\%)$ & $32(31.7)$ \\
Age, mean years \pm SD & $56.03 \pm 16.88$ \\
Poor-quality acoustic window, n (\%) & $2(2)$ \\
LVEDD-ONCO, mean cm \pm SD & $4.52 \pm 0.82$ \\
LVESD-ONCO, mean cm \pm SD & $2.75 \pm 0.70$ \\
LVEF-ONCO, mean \% \pm SD & $68.35 \pm 9.98$ \\
LVEDD-CARDIO, mean cm \pm SD & $4.77 \pm 0.79$ \\
LVESD-CARDIO, mean cm \pm SD & $2.82 \pm 0.66$ \\
LVEF-CARDIO, mean $\% \pm$ SD & $64.75 \pm 8.76$
\end{tabular}

LV, left ventricular end-diastolic diameter; LVEDD, LV end-diastolic diameter; LVEF, LV ejection fraction; LVESD, LV end-systolic diameter; ONCO, oncologist; CARDIO, cardiologist; SD, standard deviation.

the two oncologists working in the study, using a hand-held device (Mindray M7 system with a P4-2s transducer; Mindray Bio-Medical Electronics Co., Ltd., Shenzhen, China). The second was performed by a cardiologist expert in cardiovascular imaging using a 'premium' (top-of-the-line, full-feature echocardiographic system) device (Philips IE33 system with an X5-1 transducer; Philips Healthcare, Andover, MA, USA). The two echocardiograms were performed on the same day. Cardiologists were blinded to the results obtained by the oncologists.

Standard 2D and M-mode echocardiographic measurements were determined in accordance with the current American Society of Echocardiography guidelines (13). All the values were analyzed according to the same guidelines (13). Each physician completed a report, including LV end-diastolic diameter (LVEDD), LV end-systolic diameter (LVESD) and LVEF by means of the Teichholz method from M-mode acquisition.

Statistical analysis. Categorical variables were described as absolute number (\%). Continuous variables were described as mean \pm standard deviation. Kolmogorov-Smirnov test was used to assess normal distribution in continuous variables. Inter-observer agreement was evaluated by means of the intra-class correlation coefficient (ICC). Bland-Altman's plots were also constructed. A difference in LVEF $>10 \%$ between the measurement performed by the oncologist and the one performed by the cardiologist was considered to be clinically significant. The same consideration was received by those patients with an LVEF $<50 \%$ in one study and $>50 \%$ in the other one. $\mathrm{P}<0.05$ was considered to indicate a statistically significant difference. The statistical analysis was performed with SPSS PASW statistics 15.0 package (SPSS Inc., Chicago, IL, USA).

\section{Results}

Patient characteristics. Out of the 101 enrolled patients, 32 were men $(31.7 \%)$ and the mean age was $56.03 \pm 16.88$ years. Two patients $(2 \%)$ had a poor-quality acoustic window. The mean echocardiographic characteristics based on the 
Table II. Clinically significant discrepancies between the results of the echocardiograms performed by cardiologists and oncologists.

\begin{tabular}{lcccc}
\hline Characteristics & Global, n $(\%)$ & First third, n $(\%)$ & Second third, n (\%) & Final third, n $(\%)$ \\
\hline LVEF difference $>10 \%$ & $23(22.8)$ & $10(30.3)$ & $11(33.3)$ & $2(5.7)$ \\
LVEF $<$ or $>50 \%$ & $1(1.0)$ & $1(3.0)$ & $0(0.0)$ & $0(0.0)$ \\
\hline
\end{tabular}

Left ventricular ejection fraction (LVEF) difference $>10 \%$ is a difference in LVEF $>10 \%$ between the measurement performed by the oncologist and the one performed by the cardiologist. LVEF < or $>50 \%$ is LVEF inferior to $50 \%$ in one study (performed by oncologist or cardiologist) and superior to $50 \%$ in the study.

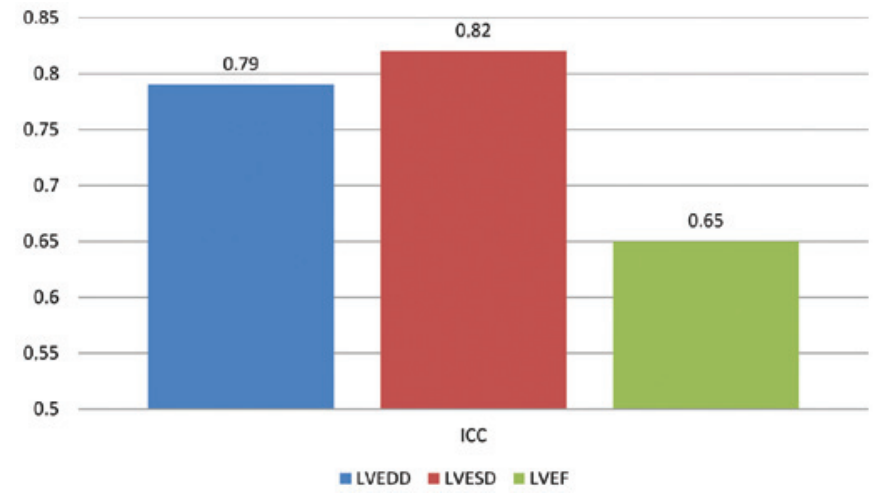

Figure 1. Intra-class correlation coefficient (ICC) for left ventricular end-diastolic diameter (LVEDD), LV end-systolic diameter (LVESD) and LV ejection fraction (LVEF) in the whole study population.

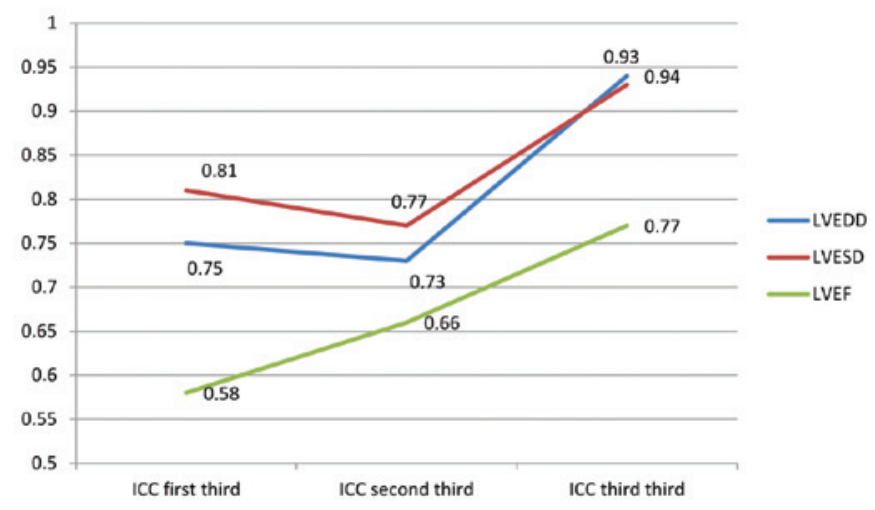

Figure 2. Evolution of the intra-class correlation coefficient (ICC) for left ventricular end-diastolic diameter (LVEDD), LV end-systolic diameter (LVESD) and LV ejection fraction (LVEF) in the three periods of the study.

measurements performed by oncologists and cardiologists are detailed in Table I.

Global agreement analysis. Agreement analysis is depicted in Fig. 1. There was a good global agreement for the three mean analyzed variables. ICC for LVEDD was $0.79,0.82$ for LVESD and 0.65 for LVEF. These data were obtained for the whole population, not taking into account the period of time when each study was performed.

Agreement analysis based on temporal division. When the results were analysed depending on the period of time when the echo studies were performed, a clear learning curve was observed. For LVEDD, ICC increased from 0.75 to 0.73 and to 0.94 along the three periods of time. For LVESD, ICC progressed from 0.81 to 0.77 and to 0.93 . Finally, LVEF started at $\mathrm{ICC}=0.58$, increased to 0.66 and in the third period of time reached 0.77 . These results and its temporal evolution are shown in Fig. 2. Bland-Altman's graphs are also depicted in Figs. 3 and 4.

Clinically significant discrepancies. As previously defined, a clinically significant difference was considered to be present if the difference in LVEF was $>10 \%$ between the measurement performed by the oncologist and the one performed by the cardiologist or the LVEF was $<50 \%$ in one study (performed by oncologist or cardiologist) and $>50 \%$ in the other one. Results are shown in Table II. In these results, a clear learning curve is also evident, particularly in the results for a difference in LVEF $>10 \%$. The number of clinically significant discrepancies was extremely low (22.8 and 1\%, respectively for the two considered variables) and they were identified only during the initial period of time.

\section{Discussion}

The present study shows for the first time that there is a good concordance between the use of a hand-held echocardiographic device by a breast cancer medical oncologist and a 'premium' system by a cardiologist for the simple, but highly important, evaluations in patients under chemotherapy, mainly the evaluation of LVEF. Therefore, these results show the possibility of limited and focused echocardiographic studies, known as echoscopic studies, to be performed by physicians different to the cardiologist. Thus, these results show that following a short training, an oncologist may be able to obtain echocardiographic images and evaluate and measure them in order to take clinical decisions on the patients regarding the use or maintenance of chemotherapy drugs.

Currently, the best tool for the follow-up appears to be echocardiography. It has the advantage of being accessible and innocuous, but a recognizable decrease in LVEF is when damage has already occurred, and with a normal LVEF it is not possible to reject the possibility of cardiotoxicity absolutely. Training should be a 'must' for any physician who is required to perform echocardiographic studies. The training period for the oncologist, according to the present results, may consist of a first one-week theoretical-practical period followed by a 4-month practical period, tailored by an expert cardiologist. During this practical period, the cardiologist should be 

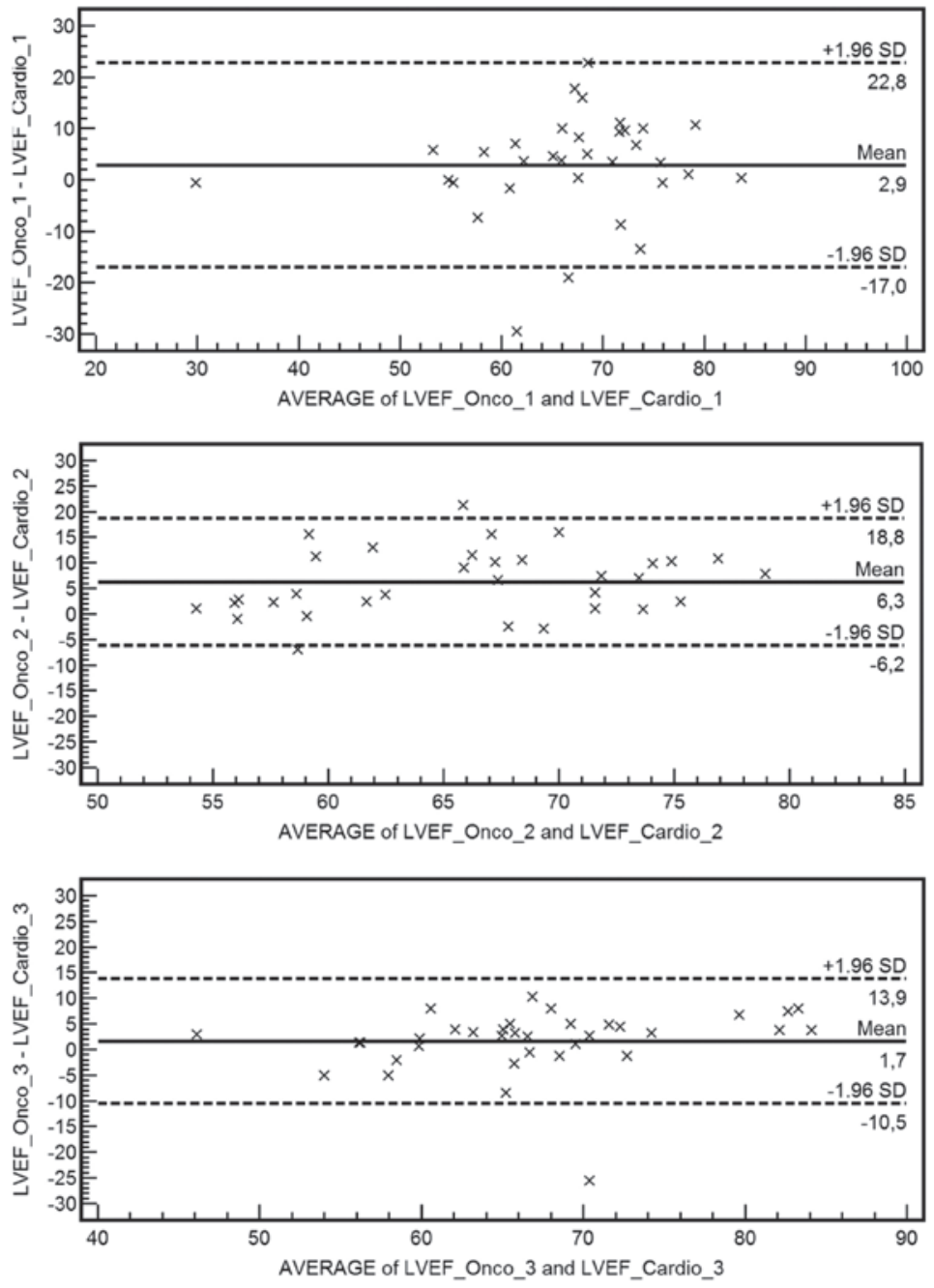

Figure 3. Bland-Altman's plot (oncologist versus cardiologist results) for left ventricular ejection fraction (LVEF) in the three periods of the study (top is the first third; middle is the second third; bottom is the final third). SD, standard deviation.
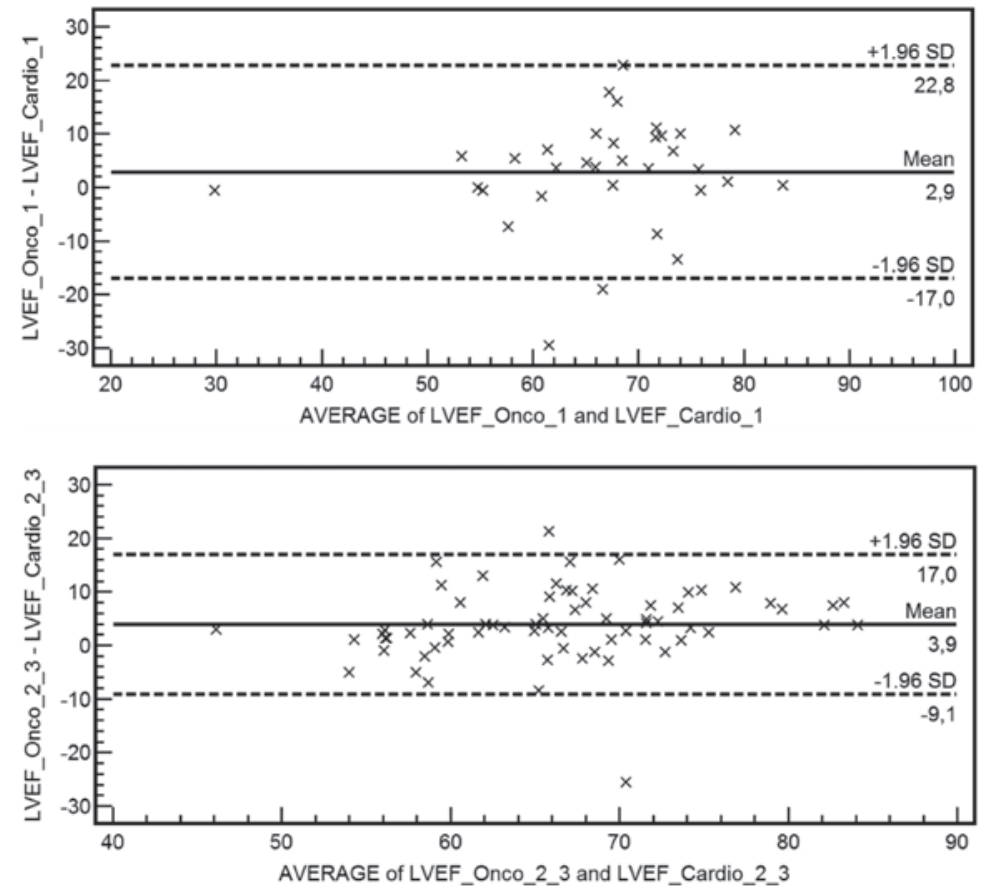

Figure 4. Bland-Altman's plot (oncologist versus cardiologist results) for left ventricular ejection fraction (LVEF) in the three periods of the study (top is the first third; bottom is the second and final thirds). SD, standard deviation. 
accessible to the oncologist to consult any doubt. This recommendation is based on the fact that, in the present study, the agreement between cardiologists and oncologists considerably increases after 4 months of practice, reaching a good level of agreement and a considerably low level of clinically significant discrepancies, as is shown in Figs. 2-4.

Previous experiences with hand-held echocardiographic systems have shown that these devices may improve the cost-effectiveness of the service provided by the echo laboratory and avoid patient discomfort derived from prolonged waiting times prior and subsequent to the examination (9). Other studies show that the use of hand-held echo systems improves workflow in the echo laboratory, avoiding the requirement for porters, patient transfer and waiting times (10-12). Furthermore, the cardiovascular imaging units should perform a large number of advanced examinations, such as stress echocardiograms and transesophageal echocardiograms. A reduction in the workload could increase the time slots dedicated to this type of examination.

The present results demonstrate that echoscopy in an oncology department may be as accurate as a conventional echocardiographic examination for a targeted evaluation. Furthermore, it may reduce the requirement for porters and the required patient time. This fact has important economic implications. Badano et al (14) published the improvement in the cost-effectiveness of the service provided by the echo laboratory for inpatients. Performing the studies in the hospital ward instead of in the echo laboratory avoids a long waiting time for patients in the echo laboratory prior and subsequent to the examination, decreases the number of days waiting for the examination and increases sonographer and echo laboratory productivity. All these improvements translate into a reduced cost of echocardiograms by $29 \%$ (14).

Thus, these hand-held devices in the hands of well-trained oncologists, may became a new standard in the evaluation of oncology patients, avoiding unnecessary waiting times and reducing the overload in the echo laboratorys.

The present study had certain limitations. The capabilities of the echo-portable devices are inferior to that of standard complete systems. Therefore, echoscopic evaluations should not replace conventional studies performed in the echo laboratory, although it is sufficient in focused evaluations (1). Of note, new prognostic markers have appeared for this type of patients and they are not evaluated by these basic studies (15). Furthermore, a learning period to acquire skills in obtaining and measuring the echocardiographic images is strongly recommended and it is necessary to provide high-quality instructions. Finally, an expert cardiologist should always be available to aid in case of doubts or technical limitations.

In conclusion, heart echoscopy performed by an oncologist with a portable device may lead to a similar clinical management as a study performed by an expert cardiologist with a 'premium' system in patients undergoing chemotherapy after a relatively short training period. These results may lead to a decrease in the echo laboratorys waiting lists and reduce patient discomfort and echo-derived costs.

\section{References}

1. Quiles J, García-Fernández MÁ, Almeida PB, Pérez-David E, Bermejo J, Moreno M and Avanzas P: Portable spectral Doppler echocardiographic device: Overcoming limitations. Heart 89: 1014-1018, 2003

2. Fiúza M: Cardiotoxicity associated with trastuzumab treatment of HER 2+ breast cancer. Adv Ther 26 (Suppl 1): S9-S17, 2009.

3. Chen MH, Kerkelä R and Force T: Mechanisms of cardiac dysfunction associated with tyrosine kinase inhibitor cancer therapeutics. Circulation 118: 84-95, 2008.

4. Bria E, Cuppone F, Milella M, Verma S, Carlini P, Nisticò C, Vaccaro V, Rossi A, Tonini G, Cognetti F, et al: Trastuzumab cardiotoxicity: Biological hypotheses and clinical open issues. Expert Opin Biol Ther 8: 1963-1971, 2008.

5. Costa RB, Kurra G, Greenberg L and Geyer CE: Efficacy and cardiac safety of adjuvant trastuzumab-based chemotherapy regimens for HER2-positive early breast cancer. Ann Oncol 21: 2153-2160, 2010.

6. Hong RA, Iimura T, Sumida $\mathrm{KN}$ and Eager RM: Cardio-oncology/onco-cardiology. Clin Cardiol 33: 733-737, 2010.

7. Martín M, Esteva FJ, Alba E, Khandheria B, Pérez-Isla L, García-Sáenz JA, Márquez A, Sengupta P and Zamorano J: Minimizing cardiotoxicity while optimizing treatment efficacy with trastuzumab: Review and expert recommendations. Oncologist 14: 1-11, 2009.

8. Murray LJ, Ramakrishnan S, O'Toole L, Manifold IH, Purohit OP and Coleman RE: Adjuvant trastuzumab in routine clinical practice and the impact of cardiac monitoring guidelines on treatment delivery. Breast 19: 339-344, 2010.

9. Spencer KT, Anderson AS, Bhargava A, Bales AC, Sorrentino M, Furlong K and Lang RM: Physician-performed point-of-care echocardiography using a laptop platform compared with physical examination in the cardiovascular patient. J Am Coll Cardiol 37: 2013-2018, 2001.

10. Kawai J, Tanabe K, Yagi T, Fujii Y, Konda T, Sumida T, Okada M, Yamaguchi K, Tani T, Yamabe K, et al: Assessment of the clinical feasibility of OptiGo for hand-held echocardiography. J Cardiol 41: 81-89, 2003 (In Japanese).

11. Xie T, Chamoun AJ, McCulloch M, Tsiouris N, Birnbaum Y and Ahmad M: Rapid screening of cardiac patients with a miniaturized hand-held ultrasound imager-comparisons with physical examination and conventional two-dimensional echocardiography. Clin Cardiol 27: 241-245, 2004.

12. Garcia Fernandez MA: Is it possible to train non-cardiologists to perform echocardiography? Rev Esp Cardiol (Engl Ed) 67: 168-170, 2014.

13. Lang RM, Bierig M, Devereux RB, Flachskampf FA, Foster E, Pellikka PA, Picard MH, Roman MJ, Seward J, Shanewise JS, et al; Chamber Quantification Writing Group; American Society of Echocardiography's Guidelines and Standards Committee; European Association of Echocardiography: Recommendations for chamber quantification: A report from the American Society of Echocardiography's Guidelines and Standards Committee and the Chamber Quantification Writing Group, developed in conjunction with the European Association of Echocardiography, a branch of the European Society of Cardiology. J Am Soc Echocardiogr 18: 1440-1463, 2005.

14. Badano LP, Nucifora G, Stacul S, Gianfagna P, Pericoli M, Del Mestre L, Buiese S, Compassi R, Tonutti G, Di Benedetto L, et al: Improved workflow, sonographer productivity and cost-effectiveness of echocardiographic service for inpatients by using miniaturized systems. Eur J Echocardiogr 10: 537-542, 2009.

15. PlanaJC,Galderisi M,Barac A,EwerMS,KyB,Scherrer-Crosbie M, Ganame J, Sebag IA, Agler DA, Badano LP, et al: Expert consensus for multimodality imaging evaluation of adult patients during and after cancer therapy: A report from the American Society of Echocardiography and the European Association of Cardiovascular Imaging. J Am Soc Echocardiogr 27: 911-939, 2014. 